\title{
Psychobiotics and the gut-brain axis: in the pursuit of happiness
}

\author{
This article was published in the following Dove Press journal: \\ Neuropsychiatric Disease and Treatment \\ 16 March 2015 \\ Number of times this article has been viewed
}

\section{Linghong Zhou' \\ Jane A Foster ${ }^{1,2}$}

'Department of Psychiatry and Behavioural Neurosciences, McMaster University, Hamilton, ON, Canada; ${ }^{2}$ Brain-Body Institute, St Joseph's Healthcare, Hamilton, ON, Canada
Correspondence: Jane A Foster Department of Psychiatry and Behavioural Neurosciences, St Joseph's Healthcare, 50 Charlton Avenue East, T3308, Hamilton, ON L8N 4A6, Canada Tel + I 905522 II 55 ext 35993

Email jfoster@mcmaster.ca

\begin{abstract}
The human intestine houses an astounding number and species of microorganisms, estimated at more than $10^{14}$ gut microbiota and composed of over a thousand species. An individual's profile of microbiota is continually influenced by a variety of factors including but not limited to genetics, age, sex, diet, and lifestyle. Although each person's microbial profile is distinct, the relative abundance and distribution of bacterial species is similar among healthy individuals, aiding in the maintenance of one's overall health. Consequently, the ability of gut microbiota to bidirectionally communicate with the brain, known as the gut-brain axis, in the modulation of human health is at the forefront of current research. At a basic level, the gut microbiota interacts with the human host in a mutualistic relationship - the host intestine provides the bacteria with an environment to grow and the bacterium aids in governing homeostasis within the host. Therefore, it is reasonable to think that the lack of healthy gut microbiota may also lead to a deterioration of these relationships and ultimately disease. Indeed, a dysfunction in the gut-brain axis has been elucidated by a multitude of studies linked to neuropsychological, metabolic, and gastrointestinal disorders. For instance, altered microbiota has been linked to neuropsychological disorders including depression and autism spectrum disorder, metabolic disorders such as obesity, and gastrointestinal disorders including inflammatory bowel disease and irritable bowel syndrome. Fortunately, studies have also indicated that gut microbiota may be modulated with the use of probiotics, antibiotics, and fecal microbiota transplants as a prospect for therapy in microbiota-associated diseases. This modulation of gut microbiota is currently a growing area of research as it just might hold the key to treatment.
\end{abstract}

Keywords: gut microbiota, mental illness, disease, modulation, therapy, probiotics

\section{Introduction: the gut-brain axis}

Bidirectional communication between the brain and the gut has been recognized with studies in the early 19 th and 20th century showing that emotional state can alter the function of the gastrointestinal (GI) tract. ${ }^{1-3}$ One of the best examples is the work of Beaumont, an army surgeon, who was able to monitor gastric secretions through a fistula in a patient's stomach and noted an association between mood and gut function. ${ }^{1}$ Early postnatal life in mammals represents a period of bacteria colonization. Normal microbiota, also referred to as commensal microbiota, colonize the mammalian gut shortly after birth and remain there throughout life. In humans, the lower intestine contains $10^{14}-10^{15}$ bacteria, that is, there are 10-100 times more bacteria in the gut than eukaryotic cells in the human body $\left(10^{13}\right) .{ }^{4-6}$ The interactions between commensal microbiota and its host are for the most part beneficial, and the presence of commensal organisms is critical to immune function, nutrient processing, other aspects of host physiology, and to brain development and function. ${ }^{7-13}$

Our understanding of the complexity and diversity of the microbiome has flourished in the past 5 years with the development of high-throughput molecular and 
metagenomic tools that provide a detailed and comprehensive analysis of the composition and diversity of species found in the gut and other microbiota communities. ${ }^{14}$ Several bacterial phyla are represented in the gut (reviewed by Diamant et $\mathrm{al}^{15}$ ) and commensals exhibit considerable diversity, with as many as 1,000 distinct bacterial species involved. ${ }^{16}$ Further, an individual's profile of microbiota is continually influenced by genetics, age, sex, and diet. ${ }^{17,18}$ While metagenomic population approaches have shown that, in general, certain bacterial populations are shared among groups of people, ${ }^{19}$ it is important to note that detailed analyses demonstrate considerable variability in bacterial content between related and unrelated individuals. ${ }^{6,20}$ As such, the microbiota profile may be a good representation of the environmental history of the individual. This dynamic nature and the diversity of the microbiome determined to date extends far beyond what researchers expected. Overall, molecular and metagenomic studies emphasize that microbiota colonies are dynamic in structure and function.

\section{Bidirectional communication in the gut-brain axis}

Established pathways of gut-brain communication include the autonomic and enteric nervous system (ENS), the neuroendocrine system, the metabolic system, and the immune system. In the past few years, there has been a rethinking of how the central nervous system (CNS) and periphery communicate, largely due to a growing body of experimental data from animal studies focused on the microbiome. Neuroscientists are now taking notice of these novel reports that highlight the "bottom-up" influence of microbes themselves, with a number of recent studies showing that resident gut bacteria are important to CNS function. Here we highlight the neural pathways that are important to microbiota-brain communication.

\section{Autonomic system and ENS}

Gut-brain neural communication pathways include sympathetic and parasympathetic nerves and the ENS, all components of the autonomic nervous system. ${ }^{21}$ Related to the gut-brain axis, the sympathetic nervous system primarily innervates the vascular beds of the GI tract and the ENS, and secondarily innervates the lamina propria and Peyer's patches, mainly in regions of $\mathrm{T}$ lymphocytes. The role of the sympathetic nervous system in the gut-brain axis includes regulating motility, blood flow, barrier function, and immune system activation. ${ }^{22}$ Bidirectional communication via the vagus nerve, a component of the parasympathetic nervous system, is a well-established pathway for gut-brain signaling and, in recent years, has emerged as an important microbiota to brain communication pathway. ${ }^{23}$ The ENS, sometimes referred to as "the second brain" comprises intrinsic primary afferent neurons, motor neurons, and glial cells contained within the myenteric plexus and the submucosal plexus that extends along the entire length of the gut. ${ }^{21}$ The ENS plays an essential role in normal intestinal function including motility and secretion.

Microbiota-ENS interactions are a fundamental factor that influences gut-brain axis function. Evidence linking gut microbiota and the ENS is provided by recent work conducted in germ-free (GF) mice. ${ }^{24,25}$ GF mice have no commensal intestinal microbiota and as such exhibit an undeveloped immune system. ${ }^{8,26-28}$ In the past decade, significant advances to our understanding of how microbiota influence gut-brain function come from research in GF model animals. Both structural and functional abnormalities were observed in GF mice at postnatal day 3 compared to specific pathogen-free (SPF) mice and mice colonized with altered Schaedler flora that normalizes the immune system. ${ }^{24}$ The myenteric plexus of the jejunum and ileum of GF mice showed an unorganized lattice-like appearance, with fewer ganglia, and thinner nerve fibers. ${ }^{24}$ Intestinal motility, measured as frequency of spontaneous muscle contractions, was decreased in GF mice, as was the amplitude of muscle contractions in GF mice compared to SPF and altered Schaedler flora-colonized mice. ${ }^{24}$ In adult GF rats, several reports have examined intestinal myoelectric activity and enteric neuromuscular function. ${ }^{29-31}$ These studies observed altered gut function in GF rats, which was normalized by conventionalization with normal microbiota. ${ }^{29-31}$ In adult GF mice, myenteric afterhyperpolarization (AH) neurons, a subtype of intrinsic primary afferent neurons in the ENS, were shown to have reduced cell excitability and reduced duration of the inhibitory slow $\mathrm{AH}$ following a single action potential generated by a short current pulse. ${ }^{25}$ In addition, resting membrane potential and input resistance were altered in GF compared to SPF mice. ${ }^{25}$ Colonization of GF mice with SPF microbiota normalized excitability and reversed the changes on passive membrane characteristics ${ }^{25}$ demonstrating that the neuronal function of these ENS neurons is mediated by the presence of gut microbiota.

The ability of gut microbiota to influence ENS function has also been demonstrated by a series of reports examining the impact of probiotic feeding on myenteric neuronal function. In rats, feeding of Lactobacillus reuteri for 9 days increased excitability in myenteric $\mathrm{AH}$ neurons and decreased the duration of the slow $\mathrm{AH} .{ }^{32}$ Intraluminal application of 
L. reuteri in an ex vivo preparation has also been shown to directly affect intestinal motility. ${ }^{33}$ Interestingly, perfusion of an in situ preparation of mouse myenteric plexus neurons with Bifidobacterium longum-fermented medium decreased the excitability of ENS neurons, ${ }^{34,35}$ suggesting that there may be some specificity in the influence of different bacteria on ENS function.

While the ENS can operate independently from the CNS, microbiota-ENS communication also influences CNS signaling systems. ${ }^{36-38}$ Neuronal processes of ENS neurons terminate in the gut epithelial lining and can respond directly to luminal contents or indirectly to neurochemicals produced by luminal bacteria or enteroendocrine cells. ${ }^{37-39}$ One pathway implicated in microbiota-ENS-brain communication is via the vagus nerve. ${ }^{23}$ It was recently suggested that ENS neurons can activate vagal afferents and thereby influence CNS function. ${ }^{38}$ In parallel, primary vagal afferents also communicate with the CNS in response to gut hormones and regulatory peptides. ${ }^{38-41}$ Evidence for the importance of the vagus nerve in microbiota-brain communication is provided by animal studies that employ subdiaphragmatic vagotomy. Probiotic administration (Lactobacillus rhamnosus) to healthy Balb/C mice resulted in reduced anxiety- and depressive-like behaviors and long-term changes in gamma-aminobutyric acid receptor expression in the CNS. ${ }^{42}$ These effects were not observed in vagotomized Balb/C mice. ${ }^{42}$ Similarly, normalization of coiltus-induced anxiety-like behavior in AKR mice by $B$. longum was vagally mediated. ${ }^{34}$ Furthermore, in an ex vivo preparation in Swiss Webster mice, intraluminal administration of L. rhamnosus increased the firing rate of the mesenteric nerve bundle indicating activation of neural pathways by this probiotic. ${ }^{43}$ This effect was blocked by subdiaphragmatic vagotomy demonstrating a role for the vagus in the earlier mentioned probiotic response. ${ }^{43}$ Overall, these animal studies demonstrate that both ENS neurons and the vagus nerve are important components of microbiota-brain neural pathways.

\section{The gut-brain axis in disease}

The human intestine houses a tremendous number and species of gut microbiota, but understanding the ability of the gut microbiota to bidirectionally communicate with the brain in the modulation of human health is at the forefront of research examining the microbiome-gut-brain axis. ${ }^{12,13,44,45}$ At a basic level, the gut microbiota interacts with the human host in a mutualistic relationship. The host intestine provides the bacteria with rich energy resources and an anaerobic, protected environment in which to grow and the bacterium aids in governing homeostasis within the host, commonly linked with the well-being of mood, metabolism, and the GI tract itself. 12,13,44,45 Therefore, it is reasonable that the lack of healthy gut microbiota may also lead to a deterioration of these relationships and ultimately disease. Indeed, a dysfunction in the gut-brain axis has been elucidated by a multitude of studies linked to neuropsychological, metabolic, and GI disorders.

\section{Neuropsychological disorders}

It has long been understood that healthy microbiota play a critical role in human development, particularly in areas such as immune development and metabolism. ${ }^{8}$ Although in its early stages, the emerging field of human microbiome research has indicated that gut microbiota may also play an important role in influencing brain development, behavior, and mood in humans. ${ }^{46,47}$ In a recent study, healthy female volunteers consumed a fermented milk product with a mixture of probiotics, including Bifidobacterium animalis subsp. Lactis, Streptococcus thermophilus, Lactobacillus bulgaricus, and Lactococcus lactis subsp. Lactis, for 4 weeks. Results showed that probiotic consumption influenced brain activity in emotional centers in healthy individuals. ${ }^{47}$ The relationship between stress, microbiota, and behavior is an important area of investigation in the field. ${ }^{12,48}$ One study showed improved mood in healthy volunteers following 3-week consumption of a probiotic-containing milk drink that contained Lactobacillus casei Shirota. ${ }^{49}$ More recently, 30-day consumption of a probiotic mixture containing Lactobacillus helveticus and B. longum showed beneficial effects on anxiety and depressive measures and showed reduced levels of stress hormone, cortisol, in healthy volunteers. ${ }^{50}$ Interestingly, a recent study that administered prebiotics (oligosaccharides that can promote growth of beneficial commensal bacteria) to healthy volunteers resulted in lower cortisol levels at awakening and improved attention to positive stimuli compared to negative stimuli in an emotional categorization task and in an emotional recognition task. ${ }^{51}$ Overall, these studies in healthy individuals provide clear evidence of a link between microbiota and emotional processing; however, to date, clinical studies focused on microbiota in psychiatric disorders have only considered depression, hepatic encephalopathy, autism spectrum disorder (ASD), and in some cases, anxiety-related symptoms associated with other medical conditions.

Though depression is a complex chronic mood disorder associated with many factors influencing its etiology including genetics and the environment, it has recently been linked 
to alterations of the gut microbiota. ${ }^{52}$ Depression is commonly described as a mood disorder in which depressed mood and/or loss of interest or pleasure in life activities are present over a period of at least 2 weeks. In addition, it must be accompanied by at least five additional symptoms that cause clinically significant impairment in social, work, or other important areas of functioning such as significant unintentional weight loss or gain, insomnia or too much sleep, fatigue or loss of energy, feelings of worthlessness or excessive guilt, diminished ability to think or concentrate, and recurrent thoughts of death. ${ }^{53}$ It is suggested that the gut microbiota affects the brain via the humoral and neural mechanisms of the gut-brain axis, with particular attention on the vagus nerve. ${ }^{52}$ Though several links between altered gut microbiota and depression have been established in animal models, few clinical studies have been conducted. However, one study that did specifically examine microbiome alterations and depression in humans by the analysis of fecal microbiota of 37 patients diagnosed with depressive disorder compared to 18 nondepressed controls did find many significant correlations. ${ }^{54}$ The most pronounced result was a general underrepresentation of Bacteroidetes in those diagnosed with depression. More specifically, Alistipes, a genus in the phylum of Bacteroidetes was overrepresented in depressed patients. Interestingly, it has been reported in $\mathrm{Balb} / \mathrm{C}$ mice to show a marked increase after a period of stress. ${ }^{55}$ Alistipes is also overrepresented in chronic fatigue syndrome ${ }^{56}$ and in irritable bowel syndrome (IBS), ${ }^{57}$ suggesting a possible common feature in several disorders that have comorbid anxiety and depression. It has been suggested that Alistipes in particular is associated with inflammation and thus potentially linked to depression through inflammatory pathways. ${ }^{55}$ Of note, it has been shown that Alistipes levels and other gut microbiota can be modified through dietary intervention, ${ }^{58}$ which may prove as an influential intervention for depressive disorders.

Anxiety and mood disorders are often comorbid with other medical conditions and in several studies that examine comorbid anxiety, a link between microbiota and psychological symptoms is observed. For example, administration of the probiotic, L. casei Shirota, decreased anxiety in patients with chronic fatigue syndrome. ${ }^{59}$ Anxiety and depression are highly comorbid in individuals with IBS. ${ }^{60}$ In IBS patients with clinically significant anxiety, daily treatment with a prebiotic galactooligosaccharide mixture for 4 weeks reduced anxiety scores and had a significant positive impact on quality of life. ${ }^{61}$ In one study, a higher Firmicutes:Bacteroides ratio in IBS patients was correlated with clinically significant depression and anxiety. ${ }^{62}$ In a more general sense, higher anxiety scores in IBS patients and healthy controls were associated with lower fecal microbial diversity supporting a link between microbiota and psychological state. ${ }^{63}$ More studies considering the role microbiota in clinical populations with anxiety disorders, mood disorders, and comorbid psychiatric symptoms are needed.

Porto-systemic encephalopathy (PSE), also known as hepatic encephalopathy, is a neuropsychiatric syndrome associated with altered gut microbiota. Its symptoms include a fluctuating level of consciousness, confusion, and progression to coma. In severe cases, it can also lead to death. ${ }^{64}$ PSE is due to an accumulation of gut-derived toxins in the bloodstream that are normally removed by liver - thus caused by hepatocellular failure or portal-systemic venous shunting. ${ }^{64}$ Both these causes would allow the hypothesized nitrogen-containing toxins such as ammonia, believed to be generated by gut bacteria from food, to enter the bloodstream and cross through the blood-brain barrier. When these toxins cross into the brain, however, they can cause the symptoms previously mentioned with regard to PSE. PSE is diagnosed by the presence of impaired liver function and an exclusion of any alternate explanation of symptoms. ${ }^{65}$ The effects of PSE are reversible with treatments that work by suppressing the production of toxic substances in the intestine, and currently, the majority of these therapeutic options in use include prebiotics, laxatives, and antibiotics. ${ }^{65}$ Prebiotics act by directly stimulating the growth of bacterial strains potentially beneficial to hosts like Bifidobacteria and Lactobacilli, thereby reducing the number and effects of potentially more harmful toxin-producing resident microbiota. One Cochrane meta-analysis of 550 randomized participants over seven trials concluded that while it is clear that probiotics are able to reduce plasma ammonia concentration when compared with placebo or no intervention, probiotics have shown some benefit in improving clinical PSE outcomes, although further randomized clinical trials are needed. ${ }^{66}$

ASD is yet another neurodevelopmental disorder in which the role of microbiota is key and another neurodevelopmental disorder at the forefront of neuroscience and child psychiatry research. ASD encompasses neurodevelopmental disorders that are defined by behavioral observations, in particular dysfunctions in social interaction and communication skills. ${ }^{53}$ The underlying causes of ASD are not yet elucidated, but it is understood that both genetic and environmental factors play a role. ${ }^{53} \mathrm{~A}$ recent review paper considering the role of gut microbiota in the etiology of ASD found that many studies have indeed identified the microbiota composition in ASD subjects to differ from those in healthy controls. ${ }^{67}$ 
For instance, Clostridium bacteria has been found consistently to be significantly elevated in those with ASD. ${ }^{68-70}$ On the other hand, there are some conflicting trends found with regard to some other strains of gut microbiota findings. For instance, Bacteroidetes have been found both to be higher ${ }^{71}$ and lower ${ }^{72}$ in ASD subjects. Other possible markers suggesting a role for gut microbiota have been found in a study that reported higher fecal bacteria fermentation product, higher ammonia concentrations, and higher short-chain fatty acid (SCFA) levels in 23 children with ASD as compared to the 9 controls. ${ }^{73}$ Further evidence of the role of the microbiota in ASD is presented by the observation that interventions with antibiotics and probiotics have led to an improvement of behavior and communication in ASD subjects. ${ }^{74}$ Looking forward, interventions that target the microbial balance may be effective in the treatment of neuropsychiatric conditions.

\section{Metabolic disorders}

Several animal and clinical studies in the past decade have demonstrated that alterations in gut microbiota may contribute to the development of obesity. ${ }^{75}$ Differences in the diversity and composition of microbiota in obese individuals have been reported. ${ }^{76,77}$ Normal intestinal flora in humans consists of several phyla, including Firmicutes and Bacteroidetes, accounting for approximately $90 \%$ of all microbiota in the human intestine. ${ }^{78}$ There is an emerging hypothesis that gut microbiota is a modulator for the obese genotype by way of an increase in the Firmicutes/Bacteroidetes (F:B) ratio. Supporting this hypothesis, one study compared the dietary intake, fecal SCFA concentrations and gut microbial profiles in 52 lean (body mass index [BMI] under 25) and 42 overweight or obese (BMI over 25) participants, finding a positive relationship between the combination of the $\log$ (F:B) ratio and SCFAs with one's BMI. ${ }^{79}$ These results were reproduced in a separate study that recorded fecal SCFA and $\mathrm{F}: \mathrm{B}$ ratios in 11 lean and 11 overweight or obese individuals. It was similarly found that overweight or obese individuals had a higher relative Firmicutes abundance, a higher F:B ratio, and increased fecal SCFA. These results are consistent with the hypothesis that overweight or obese individuals produce more colonic SCFA than lean individuals due to the differences in colonic microbiota. ${ }^{80}$

There are several possible mechanisms related to how gut microbiota may affect metabolism, such as through the regulation of GI hormones, the production of vitamins, aid in the breakdown and digestion of nutrients, and fat storage, ${ }^{81}$ which all lead to the increased efficiency of caloric extraction from food. In particular, it is suggested that Firmicutes, whose numbers have been found to be increased in obese individuals, express genes that encode enzymes that break down otherwise indigestible dietary polysaccharides, thus increasing the host's ability to harvest more energy. SCFAs are suggested to be the mediator in this obesity epidemic as a high F:B ratio leads to excess SCFA production, leading to increased colonic energy availability, contributing to overall weight gain. ${ }^{80}$ With increased interest in this field, there is an increasing number of studies and theories dedicated to the specific mechanism by which gut microbiota may contribute to weight.

\section{Gl disorders}

Gut microbial imbalance has also been linked to many GI tract disorders. IBS is associated with alterations in the gut microbiota ${ }^{82}$ and is often comorbid with anxiety disorders. ${ }^{60,83-86}$ Syndrome (IBS) is another common GI disease affecting about $20 \%$ of persons in the developed world at some point in their lives. ${ }^{87}$ It is a chronic disorder that affects the large intestine and commonly causes cramping, abdominal pain, bloating, gas, diarrhea, and constipation. Though it is known that the muscles of the intestinal walls either contract too strongly causing gas, bloating, and diarrhea or too weakly causing slow food passage and dry stools, it is not known what directly causes these contraction changes. Though factors such as food intolerance, stress, and hormones are believed to play a role, IBS is a GI disorder most closely related to altered gut microbiota. ${ }^{62}$ In fact, a study using pyrosequencing to examine the fecal microbiota profiles of 37 IBS patients age- and sex-matched to 20 controls found that the microbial signature may be related to the clinical phenotype in a subset of IBS patients. In particular, an increase in Firmicutes-associated taxa and a depletion of Bacteroidetes-related taxa best characterizes IBS subjects from the normal population. ${ }^{62}$ Similarly, a study examining the bacterial cultures of 320 subjects found a direct link between IBS and an overgrowth microbiota. ${ }^{88}$ The study found that of those patients with IBS, $37.5 \%$ were positive for small intestine bacterial overgrowth (SIBO), compared to the less than $10 \%$ without IBS. In addition, this overgrowth of bacteria in the small intestine was more prevalent in those with the more severe diarrhea-predominant version of this disease. ${ }^{88}$ This study also found a greater number of species of bacteria in IBS patients, confirming a key role of gut microbiota in IBS. ${ }^{88}$

SIBO, as previously mentioned, is a GI disease caused by an increased number and species of bacteria in the small intestine. ${ }^{89}$ Though the overall prevalence of SIBO is unknown, 
it is thought to be substantially underdiagnosed..$^{90}$ This is largely due to the fact that the etiology of the SIBO remains unclear and the clinical symptoms vary greatly from asymptomatic to many nonspecific symptoms such as bloating, flatulence, abdominal discomfort, diarrhea, and abdominal pain, many of which overlap with the symptoms of IBD. ${ }^{89}$ In more severe cases, symptoms may also include malabsorption leading to weight loss and nutritional deficiencies, liver lesions, and skin manifestations such as rosacea. ${ }^{90}$ In a more extreme case, a severe complication known as D-lactic acidosis may occur, caused by an excessive overgrowth of lactobacilli leading nonabsorbed saccharides to be fermented into the D-isomer of lactic acid, a product that cannot be metabolized by the human body. ${ }^{90}$

The concept that long-term changes in brain circuitry and function contribute to the symptoms associated with gut disorders such as IBS is supported by research conducted by imaging scientists in patients with IBS in comparison to healthy volunteers. ${ }^{91}$ Researchers conducted functional Magnetic Resonance Imaging assessment of healthy controls and IBS patients during colon distention and showed differences in central patterns of activation. ${ }^{91}$ In particular, IBS patients showed increased activation of affective brain regions in response to painful visceral stimuli and the healthy volunteers showed greater activation of arousal and attention centers. This work demonstrates that several brain regions respond to visceral pain stimuli via gut-to-brain signaling events; however, the regions activated differ between IBS patients and healthy volunteers, which may exaggerate the pain perception in patient populations. ${ }^{91}$

\section{Modulation of microbiota and prospects for therapy}

Understanding the important role that gut microbiota play in health, it is reassuring that gut microbiota can indeed be modulated as a prospect for therapy in a variety of microbiota-associated diseases. Though the use of probiotics and antibiotics has been used extensively in animal studies with regard to neuropsychological disorders such as anxiety and depression, there is limited research concerning their effects in clinical populations. However, there is increasing evidence that probiotics may be beneficial by reducing depressive and anxiety-like symptoms. For example, it was found, in a double-blind, randomized clinical trial, that healthy subjects who were given a mixture of probiotics, containing $L$. helveticus and B. longum, for 30 days demonstrated significantly less psychological distress, as measured by various questionnaires, than their matched counterparts on placebo control. ${ }^{50}$ Similarly, another double-blind, placebo-controlled trial, demonstrated that healthy subjects who scored in the lower third for depressed mood showed significant improvement, after being fed a probiotic-containing milk drink for 3 weeks, as compared to their counterparts on placebo control. ${ }^{92} \mathrm{Gut}$ microbiota alterations have also been reported in a substantial number of children with autism, particularly citing a 10-fold increase in Clostridium numbers as well as greater diversity. ${ }^{74}$ It is thought that the administration of probiotic bacteria to address changes in the microbiota may be able to reduce inflammation, restore epithelial barrier function, and potentially ameliorate behavioral symptoms associated in children with autism. ${ }^{74}$ Though the clinical studies linking the therapeutic impact of probiotics on neuropsychological disorders are limited in the early stages, their results support those from preclinical studies, suggesting that the modulation of microbiota may indeed be targeted for their therapeutic potential in neuropsychological disorders.

It has also been reported that altered gut microbiota proportions may contribute to the development of obesity with a number of theories and metabolic pathways proposed. The modulation of gut microbiota in overweight or obese individuals with probiotics is an attractive concept as a way to combat obesity and related disorders. ${ }^{93}$ Modulation of the gut microbiota can be carried out by various methods including probiotics, antibiotics, and fecal microbiota transplant. Modulation of microbiota by probiotics has proven effective in both preclinical and clinical trials - in diet-induced obese mice, those who were treated with L. curvatus and L. plantarum showed reduced weight gain as well as downregulation of proinflammatory genes in adipose tissue. ${ }^{94}$ A study in healthy individuals with a large amount of visceral fat showed that consuming fermented milk containing Lactobacillus gasseri for 12 weeks was associated with a reduction in BMI, although cessation of consumption lead to partial reversal of effects, suggesting that continuous consumption may be required to maintain beneficial effects. ${ }^{95}$ Both these studies demonstrate the potential benefits of probiotics in obesity treatment. Antibiotics such as vancomycin have also proved effective. When introduced in mice, vancomycin has significantly reduced the proportions of Firmicutes and Bacteroidetes and increased Proteobacteria, resulting in reduced weight gain and improved fasting glucose and triglyceride levels. ${ }^{96}$ Gut microbiota transplants through fecal microbiota transplants may also prove effective - in a small study infusion of microbiota from lean donors to patients with metabolic syndrome increased their insulin sensitivity when measured at 6 weeks after infusion, aiding in weight loss. ${ }^{97}$ 
Interestingly, the modulation of microbiota has been suggested as preventative against the common cold and influenza-like symptoms in children. ${ }^{98}$ In a double-blind, placebo-controlled study, 326 children aged between 3 years and 5 years were randomly assigned to receive Lactobacillus acidophilus $(\mathrm{n}=110)$, L. acidophilus and B. animalis lactis $B i-07(\mathrm{n}=112)$, or a placebo $(\mathrm{n}=104)$, treated twice daily for 6 months. At the end of the study, it was found that relative to the placebo group, those who received single and combination probiotics reduced their incidences and duration of fever, coughing, and rhinorrhea significantly. ${ }^{98}$

\section{Concluding statement}

The more the research reveals, the greater the affect gut microbiota appears to have, at nearly all systems and levels of the human body. Not surprisingly, these clinical diseases further illustrate the vital role played by gut microbiota in the human body, both through healthy, beneficial states and, more importantly, in altered, dysfunctional states. The aforementioned studies, which focus on the impact of gut microbiota on the host, are essential to our understanding the influence of these systems. In the absence or dysfunction of normal gut flora, a multitude of diseases may occur, shedding light on the important role maintained by the gut-brain axis.

\section{Disclosure}

The authors report no conflicts of interest in this work.

\section{References}

1. Beaumont W. Experiments and Observations on the Gastric Juice and the Physiology of Digestion. Plattsburg: F.P. Allen; 1833.

2. Cannon WB. The influence of emotional states on the functions of the alimentary canal. Am J Med Sci. 1909;137:480-487.

3. Pavlov I. The Work of Digestive Glands. London: Griffen; 1910. [English translation from Russian by W. H. Thompson].

4. Luckey TD. Introduction to intestinal microecology. Am J Clin Nutr. 1972;25(12):1292-1294

5. Savage DC. Microbial ecology of the gastrointestinal tract. Annu Rev Microbiol. 1977;31:107-133.

6. Gill SR, Pop M, Deboy RT, et al. Metagenomic analysis of the human distal gut microbiome. Science. 2006;312(5778):1355-1359.

7. Hooper LV, Wong MH, Thelin A, Hansson L, Falk PG, Gordon JI. Molecular analysis of commensal host-microbial relationships in the intestine. Science. 2001;291(5505):881-884.

8. Macpherson AJ, Harris NL. Interactions between commensal intestinal bacteria and the immune system. Nat Rev Immunol. 2004;4(6): 478-485.

9. Macpherson AJ, Martinic MM, Harris N. The functions of mucosal $\mathrm{T}$ cells in containing the indigenous commensal flora of the intestine. Cell Mol Life Sci. 2002;59(12):2088-2096.

10. Macpherson AJ, Uhr T. Compartmentalization of the mucosal immune responses to commensal intestinal bacteria. Ann N Y Acad Sci. 2004; 1029:36-43.

11. Tlaskalová-Hogenová H, Stepánková R, Hudcovic T, et al. Commensal bacteria (normal microflora), mucosal immunity and chronic inflammatory and autoimmune diseases. Immunol Lett. 2004;93(2-3):97-108.
12. Cryan JF, Dinan TG. Mind-altering microorganisms: the impact of the gut microbiota on brain and behaviour. Nat Rev Neurosci. 2012; 13(10):701-712.

13. Foster JA, McVey Neufeld KA. Gut-brain axis: how the microbiome influences anxiety and depression. Trends Neurosci. 2013;36(5):305-312.

14. Di Bella JM, Bao Y, Gloor GB, Burton JP, Reid G. High throughput sequencing methods and analysis for microbiome research. J Microbiol Methods. 2013;95(3):401-414.

15. Diamant M, Blaak EE, de Vos WM. Do nutrient-gut-microbiota interactions play a role in human obesity, insulin resistance and type 2 diabetes? Obes Rev. 2011;12(4):272-281.

16. Qin J, Li R, Raes J, et al; MetaHIT Consortium. A human gut microbial gene catalogue established by metagenomic sequencing. Nature. 2010;464(7285):59-65.

17. Jumpertz R, Le DS, Turnbaugh PJ, et al. Energy-balance studies reveal associations between gut microbes, caloric load, and nutrient absorption in humans. Am J Clin Nutr. 2011;94(1):58-65.

18. Kau AL, Ahern PP, Griffin NW, Goodman AL, Gordon JI. Human nutrition, the gut microbiome and the immune system. Nature. 2011; 474(7351):327-336.

19. Arumugam M, Raes J, Pelletier E, et al. Enterotypes of the human gut microbiome. Nature. 2011;473(7346):174-180.

20. Costello EK, Lauber CL, Hamady M, Fierer N, Gordon JI, Knight R. Bacterial community variation in human body habitats across space and time. Science. 2009;326(5960):1694-1697.

21. Furness JB. The enteric nervous system and neurogastroenterology. Nat Rev Gastroenterol Hepatol. 2012;9(5):286-294.

22. Cervi AL, Lukewich MK, Lomax AE. Neural regulation of gastrointestinal inflammation: role of the sympathetic nervous system. Auton Neurosci. 2014;182:83-88.

23. Forsythe P, Bienenstock J, Kunze WA. Vagal pathways for microbiomebrain-gut axis communication. Adv Exp Med Biol. 2014;817:115-133.

24. Collins J, Borojevic R, Verdu EF, Huizinga JD, Ratcliffe EM. Intestinal microbiota influence the early postnatal development of the enteric nervous system. Neurogastroenterol Motil. 2014;26(1):98-107.

25. McVey Neufeld KA, Mao YK, Bienenstock J, Foster JA, Kunze WA. The microbiome is essential for normal gut intrinsic primary afferent neuron excitability in the mouse. Neurogastroenterol Motil. 2013;25(2): e183-e188.

26. Boman HG. Innate immunity and the normal microflora. Immunol Rev. 2000;173:5-16.

27. Macpherson AJ, Uhr T. Induction of protective IgA by intestinal dendritic cells carrying commensal bacteria. Science. 2004;303(5664): $1662-1665$.

28. Tlaskalová-Hogenová H, Tucková L, Stepánková R, et al. Involvement of innate immunity in the development of inflammatory and autoimmune diseases. Ann N Y Acad Sci. 2005;1051:787-798.

29. Caenepeel P, Janssens J, Vantrappen G, Eyssen H, Coremans G. Interdigestive myoelectric complex in germ-free rats. Dig Dis Sci. 1989; 34(8):1180-1184.

30. Husebye E, Hellstrom PM, Midtvedt T. Intestinal microflora stimulates myoelectric activity of rat small intestine by promoting cyclic initiation and aboral propagation of migrating myoelectric complex. Dig Dis Sci. 1994;39(5):946-956.

31. Husebye E, Hellstrom PM, Sundler F, Chen J, Midtvedt T. Influence of microbial species on small intestinal myoelectric activity and transit in germ-free rats. Am J Physiol Gastrointest Liver Physiol. 2001;280(3): G368-G380.

32. Kunze WA, Mao YK, Wang B, et al. Lactobacillus reuteri enhances excitability of colonic AH neurons by inhibiting calcium dependent potassium channel opening. J Cell Mol Med. 2009;13(8B):2261-2270.

33. Wang B, Mao YK, Diorio C, et al. Luminal administration ex vivo of a live Lactobacillus species moderates mouse jejunal motility within minutes. FASEB J. 2010;24(10):4078-4088.

34. Bercik P, Park AJ, Sinclair D, et al. The anxiolytic effect of Bifidobacterium longum $\mathrm{NCC} 3001$ involves vagal pathways for gut-brain communication. Neurogastroenterol Motil. 2011;23(12):1132-1139. 
35. Khoshdel A, Verdu EF, Kunze W, McLean P, Bergonzelli G, Huizinga JD. Bifidobacterium longum NCC3001 inhibits AH neuron excitability. Neurogastroenterol Motil. 2013;25(7):e478-e484.

36. Bayliss WM, Starling EH. The movements and innervation of the small intestine. J Physiol. 1899;24(2):99-143.

37. Kunze WA, Bornstein JC, Furness JB. Identification of sensory nerve cells in a peripheral organ (the intestine) of a mammal. Neuroscience. 1995;66(1):1-4.

38. Perez-Burgos A, Mao YK, Bienenstock J, Kunze WA. The gut-brain axis rewired: adding a functional vagal nicotinic "sensory synapse". FASEB J. 2014;28(7):3064-3074.

39. Dockray GJ. Enteroendocrine cell signalling via the vagus nerve. Curr Opin Pharmacol. 2013;13(6):954-958.

40. Hara H, Haga S, Aoyama Y, Kiriyama S. Short-chain fatty acids suppress cholesterol synthesis in rat liver and intestine. J Nutr. 1999;129(5): 942-948.

41. Raybould HE. Gut chemosensing: interactions between gut endocrine cells and visceral afferents. Auton Neurosci. 2010;153(1-2):41-46.

42. Bravo JA, Forsythe $P$, Chew MV, et al. Ingestion of Lactobacillus strain regulates emotional behavior and central GABA receptor expression in a mouse via the vagus nerve. Proc Natl Acad Sci U S A. 2011;108(38): 16050-16055.

43. Perez-Burgos A, Wang B, Mao YK, et al. Psychoactive bacteria Lactobacillus rhamnosus (JB-1) elicits rapid frequency facilitation in vagal afferents. Am J Physiol Gastrointest Liver Physiol. 2013;304(2): G211-G220.

44. Luna RA, Foster JA. Gut brain axis: diet microbiota interactions and implications for modulation of anxiety and depression. Curr Opin Biotechnol. 2014;32C:35-41.

45. Rhee SH, Pothoulakis C, Mayer EA. Principles and clinical implications of the brain-gut-enteric microbiota axis. Nature reviews. Nat Rev Gastroenterol Hepatol. 2009;6(5):306-314.

46. Mayer EA, Knight R, Mazmanian SK, Cryan JF, Tillisch K. Gut microbes and the brain: paradigm shift in neuroscience. $J$ Neurosci. 2014;34(46):15490-15496.

47. Tillisch K, Labus J, Kilpatrick L, et al. Consumption of fermented milk product with probiotic modulates brain activity. Gastroenterology. 2013;144(7):e1391-e1394.

48. Dinan TG, Cryan JF. Regulation of the stress response by the gut microbiota: implications for psychoneuroendocrinology. Psychoneuroendocrinology. 2012;37(9):1369-1378.

49. Benton D, Williams C, Brown A. Impact of consuming a milk drink containing a probiotic on mood and cognition. Eur J Clin Nutr. 2007;61(3): $355-361$.

50. Messaoudi M, Lalonde R, Violle N, et al. Assessment of psychotropiclike properties of a probiotic formulation (Lactobacillus helveticus R0052 and Bifidobacterium longum R0175) in rats and human subjects. Br J Nutr. 2011;105(5):755-764.

51. Schmidt K, Cowen PJ, Harmer CJ, Tzortzis G, Errington S, Burnet PW. Prebiotic intake reduces the waking cortisol response and alters emotional bias in healthy volunteers. Psychopharmacology (Berl). Epub 2014 Dec 3.

52. Dash S, Clarke G, Berk M, Jacka FN. The gut microbiome and diet in psychiatry: focus on depression. Curr Opin Psychiatry. 2015;28(1):1-6.

53. American Psychiatric Association; American Psychiatric Association; DSM-5 Task Force. Diagnostic and Statistical Manual of Mental Disorders: DSM-5. 5th ed. Washington, D.C: American Psychiatric Association; 2013.

54. Naseribafrouei A, Hestad K, Avershina E, et al. Correlation between the human fecal microbiota and depression. Neurogastroenterol Motil. 2014;26(8):1155-1162.

55. Bangsgaard Bendtsen KM, Krych L, Sørensen DB, et al. Gut microbiota composition is correlated to grid floor induced stress and behavior in the BALB/c mouse. PLoS One. 2012;7(10):e46231.

56. Fremont M, Coomans D, Massart S, De Meirleir K. High-throughput 16S rRNA gene sequencing reveals alterations of intestinal microbiota in myalgic encephalomyelitis/chronic fatigue syndrome patients. Anaerobe. 2013;22:50-56.
57. Saulnier DM, Riehle K, Mistretta TA, et al. Gastrointestinal microbiome signatures of pediatric patients with irritable bowel syndrome. Gastroenterology. 2011;141(5):1782-1791.

58. David LA, Maurice CF, Carmody RN, et al. Diet rapidly and reproducibly alters the human gut microbiome. Nature. 2014;505(7484):559-563.

59. Rao AV, Bested AC, Beaulne TM, et al. A randomized, double-blind, placebo-controlled pilot study of a probiotic in emotional symptoms of chronic fatigue syndrome. Gut Pathog. 2009;1(1):6.

60. Fond G, Loundou A, Hamdani N, et al. Anxiety and depression comorbidities in irritable bowel syndrome (IBS): a systematic review and metaanalysis. Eur Arch Psychiatry Clin Neurosci. 2014;264(8):651-660.

61. Silk DB, Davis A, Vulevic J, Tzortzis G, Gibson GR. Clinical trial: the effects of a trans-galactooligosaccharide prebiotic on faecal microbiota and symptoms in irritable bowel syndrome. Aliment Pharmacol Ther. 2009;29(5):508-518.

62. Jeffery IB, O'Toole PW, Öhman L, et al. An irritable bowel syndrome subtype defined by species-specific alterations in faecal microbiota. Gut. 2012;61(7):997-1006.

63. Sundin J, Rangel I, Kumawat AK, Hultgren-Hornquist E, Brummer RJ. Aberrant mucosal lymphocyte number and subsets in the colon of postinfectious irritable bowel syndrome patients. Scand J Gastroenterol. 2014;49(9):1068-1075.

64. Cash WJ, McConville P, McDermott E, McCormick PA, Callender ME, McDougall NI. Current concepts in the assessment and treatment of hepatic encephalopathy. QJM. 2010;103(1):9-16.

65. Garcovich M, Zocco MA, Roccarina D, Ponziani FR, Gasbarrini A. Prevention and treatment of hepatic encephalopathy: focusing on gut microbiota. World J Gastroenterol. 2012;18(46):6693-6700.

66. McGee RG, Bakens A, Wiley K, Riordan SM, Webster AC. Probiotics for patients with hepatic encephalopathy. Cochrane Database Syst Rev. 2011;11:CD008716.

67. Louis P. Does the human gut microbiota contribute to the etiology of autism spectrum disorders? Dig Dis Sci. 2012;57(8):1987-1989.

68. Finegold SM, Molitoris D, Song Y, et al. Gastrointestinal microflora studies in late-onset autism. Clin Infect Dis. 2002;35(suppl 1): S6-S16.

69. Parracho HM, Bingham MO, Gibson GR, McCartney AL. Differences between the gut microflora of children with autistic spectrum disorders and that of healthy children. $J$ Med Microbiol. 2005;54(pt 10): 987-991.

70. Song Y, Liu C, Finegold SM. Real-time PCR quantitation of clostridia in feces of autistic children. Appl Environ Microbiol. 2004;70(11): 6459-6465.

71. Wang L, Christophersen CT, Sorich MJ, Gerber JP, Angley MT, Conlon MA. Low relative abundances of the mucolytic bacterium Akkermansia muciniphila and Bifidobacterium spp. in feces of children with autism. Appl Environ Microbiol. 2011;77(18):6718-6721.

72. Williams BL, Hornig M, Buie T, et al. Impaired carbohydrate digestion and transport and mucosal dysbiosis in the intestines of children with autism and gastrointestinal disturbances. PLoS One. 2011;6(9): e24585.

73. Wang L, Christophersen CT, Sorich MJ, Gerber JP, Angley MT, Conlon MA. Elevated fecal short chain fatty acid and ammonia concentrations in children with autism spectrum disorder. Dig Dis Sci. 2012; 57(8):2096-2102.

74. Critchfield JW, van Hemert S, Ash M, Mulder L, Ashwood P. The potential role of probiotics in the management of childhood autism spectrum disorders. Gastroenterol Res Pract. 2011;2011:161358.

75. Clarke SF, Murphy EF, O'Sullivan O, et al. Targeting the microbiota to address diet-induced obesity: a time dependent challenge. PLoS One. 2013;8(6):e65790.

76. Bäckhed F, Ding H, Wang T, et al. The gut microbiota as an environmental factor that regulates fat storage. Proc Natl Acad Sci U S A. 2004; 101(44):15718-15723.

77. Ley RE, Backhed F, Turnbaugh P, Lozupone CA, Knight RD, Gordon JI. Obesity alters gut microbial ecology. Proc Natl Acad Sci U S A. 2005; 102(31):11070-11075. 
78. Eckburg PB, Bik EM, Bernstein CN, et al. Diversity of the human intestinal microbial flora. Science. 2005;308(5728):1635-1638.

79. Fernandes J, Su W, Rahat-Rozenbloom S, Wolever TM, Comelli EM. Adiposity, gut microbiota and faecal short chain fatty acids are linked in adult humans. Nutr Diabetes. 2014;4:e121.

80. Rahat-Rozenbloom S, Fernandes J, Gloor GB, Wolever TM. Evidence for greater production of colonic short-chain fatty acids in overweight than lean humans. Int J Obes. 2014;38(12):1525-1531.

81. Cani PD, Delzenne NM. The gut microbiome as therapeutic target. Pharmacol Ther. 2011;130(2):202-212.

82. De Palma G, Collins SM, Bercik P, Verdu EF. The microbiota-gut-brain axis in gastrointestinal disorders: stressed bugs, stressed brain or both? J Physiol. 2014;592(pt 14):2989-2997.

83. Whitehead WE, Palsson O, Jones KR. Systematic review of the comorbidity of irritable bowel syndrome with other disorders: what are the causes and implications? Gastroenterology. 2002;122(4):1140-1156.

84. Sartor RB. Mechanisms of disease: pathogenesis of Crohn's disease and ulcerative colitis. Nature clinical practice. Gastroenterol Hepatol. 2006;3(7):390-407.

85. Gevers D, Kugathasan S, Denson LA, et al. The treatment-naive microbiome in new-onset Crohn's disease. Cell Host Microbe. 2014;15(3): 382-392.

86. Andoh A, Kobayashi T, Kuzuoka H, et al. Characterization of gut microbiota profiles by disease activity in patients with Crohn's disease using data mining analysis of terminal restriction fragment length polymorphisms. Biomed Rep. 2014;2(3):370-373.

87. Torpy JM, Golub RM. JAMA patient page. Irritable bowel syndrome. JAMA. 2011;306(13):1501.

88. Kim G, Deepinder F, Morales W, et al. Methanobrevibacter smithii is the predominant methanogen in patients with constipation-predominant IBS and methane on breath. Dig Dis Sci. 2012;57(12):3213-3218.
89. Khoshini R, Dai SC, Lezcano S, Pimentel M. A systematic review of diagnostic tests for small intestinal bacterial overgrowth. Dig Dis Sci. 2008;53(6):1443-1454.

90. Bures J, Cyrany J, Kohoutova D, et al. Small intestinal bacterial overgrowth syndrome. World J Gastroenterol. 2010;16(24):2978-2990.

91. Hall GB, Kamath MV, Collins S, et al. Heightened central affective response to visceral sensations of pain and discomfort in IBS. Neurogastroenterol Motil. 2010;22(3):e276-e280.

92. Barrett E, Ross RP, O'Toole PW, Fitzgerald GF, Stanton C. Gammaaminobutyric acid production by culturable bacteria from the human intestine. J Appl Microbiol. 2012;113(2):411-417.

93. Moran CP, Shanahan F. Gut microbiota and obesity: role in aetiology and potential therapeutic target. Best Pract Res Clin Gastroenterol. 2014;28(4):585-597.

94. Park DY, Ahn YT, Park SH, et al. Supplementation of Lactobacillus curvatus HY7601 and Lactobacillus plantarum KY1032 in diet-induced obese mice is associated with gut microbial changes and reduction in obesity. PLoS One. 2013;8(3):e59470.

95. Kadooka Y, Sato M, Ogawa A, et al. Effect of Lactobacillus gasseri SBT2055 in fermented milk on abdominal adiposity in adults in a randomised controlled trial. Br J Nutr. 2013;110(9):1696-1703.

96. Murphy EF, Cotter PD, Hogan A, et al. Divergent metabolic outcomes arising from targeted manipulation of the gut microbiota in diet-induced obesity. Gut. 2013;62(2):220-226.

97. Vrieze A, Van Nood E, Holleman F, et al. Transfer of intestinal microbiota from lean donors increases insulin sensitivity in individuals with metabolic syndrome. Gastroenterology. 2012;143(4): 913-916.e917.

98. Leyer GJ, Li S, Mubasher ME, Reifer C, Ouwehand AC. Probiotic effects on cold and influenza-like symptom incidence and duration in children. Pediatrics. 2009;124(2):e172-e179.
Neuropsychiatric Disease and Treatment

\section{Publish your work in this journal}

Neuropsychiatric Disease and Treatment is an international, peerreviewed journal of clinical therapeutics and pharmacology focusing on concise rapid reporting of clinical or pre-clinical studies on a range of neuropsychiatric and neurological disorders. This journal is indexed on PubMed Central, the 'PsycINFO' database and CAS,

\section{Dovepress}

and is the official journal of The International Neuropsychiatric Association (INA). The manuscript management system is completely online and includes a very quick and fair peer-review system, which is all easy to use. Visit http://www.dovepress.com/testimonials.php to read real quotes from published authors. 